Beitrag zum Themenschwerpunkt „DaF in China“

V. Kontrast von Sprache und Kommunikation

Gabriele E. OTTO*

Begegnungen von Wissenschafts- und Lernkulturen im Netz - Herausforderungen und Chancen für eine Didaktik des wissenschaftlichen Schreibens für chinesische Studierende der Germanistik in Deutschland

Encounters of Science and Learning Cultures in the Internet - Challenges and Opportunities for the Didactics of Scientific Writing for Chinese Students of German Studies in Germany

https://doi.org/10.1515/infodaf-2019-0022

Zusammenfassung: Der Beitrag wendet sich Desideraten zur Etablierung einer plurikulturellen Wissenschaftskommunikation im Kontext von Partizipationswegen von Jung-Akademiker*innen zu. Dazu wird zunächst die Wissenschaftsrezeption von Studierenden bei der Nutzung des Internets bei Seminararbeiten skizziert. Danach werden mithilfe von Wissenschaftstheorie sowie den Transferund Kulturwissenschaften wesentliche Merkmale und Bedarfe von Wissenschaftskommunikation ermittelt und dargestellt. Anschließend werden anhand von Praxisbeispielen Wege aufgezeigt, welche Aktivitäten von etablierten Wissenschaftler*innen notwendig erscheinen, um die Wissenschaftsrezeption von Studierenden im Netz ausreichend zu begleiten und anzuleiten. Dazu werden Felder des Austauschs dargestellt und in ihrem Potential zur Entwicklung einer transkulturellen Germanistik skizziert, die als Basis für kulturelles Lernen in der Wissenschaft angesehen wird.

*Kontaktperson: Gabriele E. OTTO, E-Mail: sisu-daad-otto@outlook.de 
Schlüsselwörter: Wissenschaftskommunikation, kulturelles Lernen, wissenschaftliches Schreiben, Open Access, Germanistik als Fremdsprachenphilologie

\begin{abstract}
The article addresses a perceived need to establish ways for pluri-cultural scholarly communication which will enable young academics to participate in academic communication. Initially, student approaches for using Internet sources for seminar papers are outlined from the perspective of reception of scientific texts in the field of literary studies. Essential characteristics and requirements of academic communication are then identified and described through employing theory of knowledge, transfer sciences and cultural sciences. Practical examples then propose ways for how established scientists could use the Internet to accompany and guide students in their more extensive use of recognized scientific internet sources. To this end, fields of interaction are described and their potential for developing transcultural German Studies in the Internet are proposed as a basis of cultural learning for science and research works.
\end{abstract}

Keywords: Scientific communication, cultural learning, scientific writing, open access, German Studies as foreign language philology

\title{
0 Einleitung
}

Ausgangspunkt für die Thematisierung des Internets als Begegnungsort verschiedener Lern- und Wissenschaftskulturen sind Untersuchungsergebnisse zur Nutzung des Internets, wie sie sich in Bachelorarbeiten, aber auch in Seminararbeiten auf verschiedenen Niveaustufen der akademischen Ausbildung niederschlägt.

Gegenstand dieser Arbeit sind die Ergebnisse aus Untersuchungen innerhalb von insgesamt 30 Seminararbeiten auf dem Gebiet der Literaturwissenschaften, insbesondere der Erzählanalyse, die an der germanistischen Fakultät einer chinesischen Hochschule verfasst wurden. Dem verbreiteten Phänomen des Copy \& Paste wird noch einmal nachgegangen, allerdings verbunden mit Fragen nach Formen und Funktionen sowie etwaigen Ursachen für diese Arbeitsweise in der Texterstellung. Ziel war es, etwas über die Hürden auf dem Weg zu einer erfolgreichen Partizipation am wissenschaftlichen Diskurs in Erfahrung zu bringen und nach den Lernprozessen zu fragen, die Studierende aufgrund lernkultureller Prägungen, die in ihren Herkunftsländern präsent sind und von daher ihre Einstellungen im Umgang mit Wissen prägen, durchlaufen müssen. Offensichtlich wurde, dass der Bedarf über eine bloße Vermittlung neuer Arbeitstechniken hinausgeht und eine Art kulturellen Lernens im kulturwissenschaftlichen Sinne im 
Hinblick auf Verfahrens- und Arbeitsweisen in der Quellenrezeption und -verwertung, wie sie im internationalen Kontext erwartet werden, initiiert werden muss (vgl. Agiba 2016: 405f. und Altmayer 2004: 459f.).

Die Relevanz des Themas ergibt sich sowohl aus der steigenden Verwendung des Internets als Wissensspeicher als auch aus der Zugänglichkeit von Internetquellen für immer größere Nutzergruppen, wodurch Studierende und Forschende unterschiedlicher Lern- und Wissenschaftskulturen zusammentreffen und das Spektrum für wissenschaftliches Arbeiten nach anerkannten Formen internationaler Standards erweitert und auch herausgefordert ist.

Die Untersuchungen lassen manifest werden, dass der Zugriff auf Materialien aus dem Internet gegenüber der Bibliotheksnutzung problematische Aspekte birgt, wie es die Ergebnisse der Untersuchungen an fünf Fallbeispielen zeigen, die nachfolgend skizziert werden.

Zudem werden anhand von Neusers Ausführungen zur globalen Weltwissensgesellschaft Bedarfe und Möglichkeiten der aktiven Partizipation im virtuellen Erfahrungs- und Handlungsraum aus wissenschaftstheoretischer Perspektive aufgezeigt und diskutiert (vgl. Neuser 2013: 330).

Differenzierte Erkenntnisse zu Prozessen des Wissenstransfers, den Rollen seiner Akteure sowie den Relationen zwischen unterschiedlichen Typen von Wissen werden den Transferwissenschaften entnommen (vgl. die gleichnamige Buchreihe, herausgegeben seit 2001 von Antos und Wichter bzw. Ballod, Roelcke und Weber). Sie dienen zur Erhellung der Handlungsmöglichkeiten in internationalen Kontexten des Wissensaustauschs. Vertiefend lassen sich die Austausch- und Aushandlungssituationen, die durch die Möglichkeiten des Internets wiederum eine erhöhte Relevanz erfahren, abschließend vor der Folie der Transkulturalität (vgl. Welsch 1997: 67ff.), aber auch aus kommunikationstheoretischer Perspektive (vgl. Schiewer 2010: 135ff.) diskutieren.

Anhand von Modellen aus der Praxis der (germanistischen) Wissenschaftskommunikation werden Fragen danach erörtert, wie etablierte Wissenschaftler*innen, die bereits Prozesse der Auseinandersetzung mit unterschiedlichen Wissenschaftskulturen durchlaufen haben, unterstützend wirken können. Welches Wissen könnte auf welche Weise bereitgestellt und verfügbar gemacht werden, um sowohl Unterschiede in den Kulturen als auch Anschlussmöglichkeiten explizit zu machen?

Modelle der gemeinsamen, auch transkulturellen Bearbeitung von Austauschsituationen werden abschließend diskutiert. 


\section{Nutzung und Herausforderung in der Arbeit mit Internetquellen}

Eine Erweiterung des Angebots an wissenschaftlichen Quellen im Internet, wie sie auf europäischer Ebene 2002/2003 mit der Budapester Initiative zur Entwicklung von Open Access-Publikationen beschlossen wurde (vgl. www.budapestopenaccessinitiative.org), ist unter verschiedenen Aspekten auch für die Arbeit in der Germanistik zu begrüßen, besonders auch in der Auslandsgermanistik, wo eine physische fortlaufend aktualisierte Bibliothek aus finanziellen oder klimatischen Gründen kaum sinnvoll zu betreiben ist. Eine deutlich höhere Internetpräsenz wissenschaftlichen Wissens käme den zunehmend thematisch breiter gefassten Forschungs- und Arbeitsinteressen der heutigen Nachwuchsgeneration trotz aller Herausforderungen entgegen.

Ziel dieses Beitrags ist, der Relation zwischen regionalen und internationalen Lern- und Wissenschaftskulturen sowie Fragen danach, welches Wissen dazu bereitgestellt werden muss, um eine bewusste Auseinandersetzung mit unterschiedlichen Kulturen gerade auch im Internet anzuleiten, nachzugehen. Zentral ist die Frage, welche Lernschritte erforderlich sind und welche Informationen über verschiedene Wissenschaftskulturen bereitgestellt werden müssen, um eine Anknüpfbarkeit zwischen unterschiedlichen Lernkulturen und den international anerkannten Wissenschaftsstandards zu erreichen.

\subsection{Art, Qualität und Funktion von Quellen - Wissensaneignung und wissenschaftliches Arbeiten}

Schaut man die Probleme beim wissenschaftlichen Arbeiten einmal genauer an, dann lassen sich im Wesentlichen zwei Problemfelder erkennen. Als Erstes ist die fehlende bzw. unzureichende Markierung von Zitaten und Quellen zu nennen, die sich entweder in einem kompletten Copy \& Paste niederschlägt oder in einer Art „Verschreibung“ des Originaltextes. Das heißt, der Text wird zwar grammatikalisch verändert, jedoch wird die Quelle als Textbasis nicht angegeben, so dass das vorliegende Produkt als Originaltext des/der jeweiligen Studierenden erscheint. Lediglich am Ende eines Absatzes, einer Seite oder erst im Quellenverzeichnis finden sich Hinweise auf die Quellen. Ein weiteres Phänomen tritt insbesondere im Kapitel Theorie und Methodik auf, wo Quellen übernommen oder teilzitiert werden, ohne dass jedoch daraus eine theoriegeleitete Methode in der Arbeit selbst angewendet wird. 
Ein zweites Problemfeld ist in den genutzten Quellen selbst zu sehen. Festzustellen ist hierbei, dass die Studierenden sich zumeist gar nicht anerkannter Fachtexte bedienen. Sie benutzen stattdessen wikipedia, grin.com, hausarbeiten. de, lerntippsammlung.de, lehrerfortbildung-bw.de und ähnliche Quellen. Was zeichnet diese Quellen aus? Sie sind in den Suchmaschinen leicht auffindbar, die Texte sind sprachlich-stilistisch eher einfach gehalten und weisen, da sie nicht an ein Fachpublikum gerichtet sind, eine deutlich geringere fachliche Intertextualität als Fachtexte auf.

Was ist neben dem Plagiatsvorwurf das Problematische an dem oben geschilderten Umgang mit den Quellen aus dem Blickwinkel des wissenschaftlichen Arbeitens? Wenn man annimmt, dass es international üblich ist, einen Gegenstand mit einer Methode zu untersuchen, die Ergebnisse zusammenzufassen und mit vorhandenen Forschungsarbeiten zu vergleichen, das heißt, die eigenen Ergebnisse im Kontext vorhandener Forschung zu diskutieren, dann führen Copy \& Paste sowie das „Verschreiben“ durch das Verwischen der Grenze zwischen eigenen Gedanken und fremden Positionen dazu, dass eine Forschungsdiskussion nicht stattfinden kann. Auch die unmarkierte Übernahme fremder Gedanken in der Phase der Erarbeitung einer Fragestellung verhindert die explizite Auseinandersetzung mit Forschungsmeinungen. Das für die Wissenschaft und ihre Fortschritte wichtige Moment der mehr oder minder kritischen Erörterung von Forschungsmeinungen verschwindet bzw. es wird auf studentischer Seite durch die geschilderte Nutzungsweise von Quellen nicht als wissenschaftliche Praxis angeeignet und trainiert, denn eigene Gedanken und Beobachtungen zum Untersuchungsgegenstand werden nicht explizit publik gemacht.

Fragen lässt sich also nach den Gründen für die Verwendung unpassender Quellen. Eine kurze Befragung von 17 chinesischen Masterstudierenden der Germanistik nach der Funktion von Zitaten in Seminararbeiten und den Gründen ihrer Verwendung gibt hier ein paar Einblicke:

Man verwende Zitate, um Copy \& Paste zu vermeiden / um die eigene Meinung zu unterstützen / wenn einem selbst nichts einfällt / um den eigenen Text zu verlängern / weil ein Autor oder eine Autorin eines wissenschaftlichen Textes, der schon veröffentlicht ist, mehr Bedeutung hat als die eigene Meinung / um Meinungen zu erörtern. (Studentische Statements)

Unter den genannten Antworten finden sich durchaus solche, die den Zweck von Zitaten in der wissenschaftlichen Praxis korrekt benennen. Drei der genannten Verwendungsgründe, nämlich, dass der Autor eines vorhandenen wissenschaftlichen Textes eine größere Bedeutung habe, weil man selbst nichts zu schreiben wisse oder lediglich, um Copy \& Paste zu vermeiden, weisen aber darauf hin, dass ein Zitat zumindest unter anderem die Funktion hat, anstelle eines eigenen Textes eingesetzt zu werden. Diese Praktiken finden sich allerdings weit häufiger in den 
untersuchten Seminararbeiten als eine explizite Auseinandersetzung mit Quellen oder auch nur die Verwendung von Zitaten zur Stützung eigener Untersuchungsergebnisse oder der Entwicklung einer Untersuchungsfrage.

Dieses Arbeitsverhalten ist umso erstaunlicher, als die Studierenden im literaturwissenschaftlichen Seminar zum Teil sehr gut im Unterrichtsgespräch die Primärquellen mithilfe eines Untersuchungsrasters der Narratologie untersuchten und auch ihre eigenen Beobachtungen am Text mit jenen, wie sie in Textrezensionen zu den ausgewählten Autoren gelesen wurden, verglichen. In der nachfolgenden Seminararbeit, der Erarbeitung einer Erzählanalyse an einer weiteren Primärquelle, wurden diese Kenntnisse jedoch häufig nicht oder nur in geringem Umfang angewandt und selbst sehr aktive Studierende ersetzten eine eigenständige Analyse und eine darauf aufbauende Interpretation durch Copy \& Paste. Dabei waren die Quellen nicht nur unter wissenschaftlichen Kriterien ungeeignet, sondern passten auch von den Inhalten her nicht zur Aufgabe der Narrationsanalyse.

\subsection{Tradiertes Verhalten im Umgang mit Quellen}

$\mathrm{Zu}$ vermuten ist daher, dass es eher eine Frage der vertrauten Praxis ist, gerade keine eigenen Texte zu produzieren, auf Vorhandenes zurückzugreifen und in Vermeidung des bloßen Referierens von Texten die Grenzen zwischen Zitat und eigener Erarbeitung unmarkiert zu lassen. Blickt man auf die Lerntraditionen in China und die vertrauten schulischen Lernpraktiken, so gilt nach wie vor das Auswendiglernen von Primär- und auch kommentierenden Sekundärquellen im muttersprachlichen Unterricht als Standard in der Beschäftigung mit literarischen Texten. ${ }^{1}$ Dies hat zweifelsohne den Vorteil, dass späterhin viele Texte als Repertoire gespeichert sind und ein Rückgriff darauf leicht möglich ist. Das Lehr-LernVerfahren begründet sich auf dem Respekt vor den tradierten Quellen. $\mathrm{Zu}$ vergleichbaren Beobachtungen im Umgang mit Quellen kommt Sändig (2009: 161) in ihrer Untersuchung zur Anfertigung literaturwissenschaftlicher Kurzessays durch fremdsprachige Studierende an der Universität Potsdam, wenn sie anmerkt, dass „Zitiertraditionen einiger asiatischer und afrikanischer Länder [...] nach der ungeschriebenen Regel funktionieren: Je verehrungswürdiger die zitierte Autorität, desto unangemessener erscheint es, das Zitat zu markieren.“ Hier deutet sich an,

1 Ich beziehe mich hier auf Auskünfte von Dozent*innen, sowie auf Beobachtungen in der Prüfungspraxis. 
dass der Umgang mit Quellen nicht einzig eine Frage von adäquater Markierung von Textstellen ist. Vielmehr werden das Selbstverständnis der Jung-Akademiker*innen, ihr Handlungsrepertoire und ihre gewohnten Handlungskompetenzen durch jene als internationale Standards gesetzte wissenschaftliche Arbeitsweisen herausgefordert. Das Aneignen und Adaptieren eigener Aktivitäten, eine auch kritische Darstellung bestehender Forschungsmeinungen berühren tiefere Schichten des Lernerselbstverständnisses. Und nicht nur dies: Die Praxis der kritischen Rezeption wissenschaftlicher Texte kann nicht in einem einzigen Seminar erlernt werden und schon gar nicht erst individuell in der Ausarbeitung der Seminararbeit vollzogen werden. Sie muss bereits Bestandteil des gesamten akademischen Lehr-Lern-Diskurses sein und somit ist auch die Lehrpraxis davon betroffen und daraus folgernd auch wiederum das Rollenverständnis der Lehrenden sowie die Lehrer/Dozenten-Studierenden-Relation.

\subsection{Praxis der Quellenverwendung und Kritik - Fallbeispiele}

Von den fünf ausgewählten und eingehender untersuchten Seminararbeiten sollen drei Fälle vorgestellt werden. $\mathrm{Zu}$ den beiden anderen Fällen werden die Beobachtungen lediglich ergänzend angeführt.

\section{Fall 3 und 4: Unmarkierte Teil-Zitate - uneindeutige Quellenangaben}

Bei diesen Fällen liegt eine ganze Reihe von Phänomenen vor, die deutliche Abweichungen von Standards des Erstellens eines Textes, der wissenschaftlichen Ansprüchen genügen soll, aufweisen. So bleiben an mehreren Stellen Zitate unmarkiert. Dies gilt zum einen für die Wiedergabe der Handlung in der Kurzgeschichte Das Brot von Wolfgang Borchert (1949/1996) (Seminararbeit S. 3). Hier wird ein Wikipedia-Text direkt verwendet, ohne ihn als Zitat zu kennzeichnen (vgl. Tab. 1, Beispiel 1). Die Quelle wird einzig im Literaturverzeichnis genannt. Der Text erscheint im Wikipedia-Original (<https://de.wikipedia.org/wiki/Das_Brot>; 01.02.2019) unter der Rubrik „Handlung“, worauf der Einleitungssatz in der Seminararbeit verweist.

Im weiteren Verlauf der Arbeit wird eine Buchquelle $\mathrm{zu}$ dem Primärwerk Streuselschnecke von Julia Franck (2002/2011) ordentlich im Zuge der Erzählanalyse der Kurzgeschichte zitiert. Allerdings lässt der das Zitat umgebende Text keinerlei Bezug zum Zitat erkennen. 
[...] Dadurch stellt sich die Frage, wer dieser Mann eigentlich ist. „Ich Erzählerin zeigt hier die Subjektivität und hat einen begrenzten Blickwinkel.“ (Schlicht/Marsch 2012, a.a. O. [Seitenzahl ist nicht angegeben]). Und die Erzählung wird aus der Rückschau geschrieben. Der Leser erlebt das Geschehen so, wie es die Ich-Erzählerin erlebt hat. Es gibt auch neutrales Erzählverhalten und sachlichen Bericht. (Seminararbeit, S. 5)

Die Erzählanalyse ist in sich nicht schlüssig. Es wird weder das im Seminar erarbeitete Analyseraster noch eine andere Systematik angewendet. Daher stellt sich die Frage, worauf die gemachten Aussagen zum Text basieren. Dies erschließt sich im Fortgang der Seminararbeit. Dort werden die beiden Kurzgeschichten Das Brot von Wolfgang Borchert und Streuselschnecke von Julia Franck hinsichtlich der Erzählgestaltung verglichen (Seminararbeit, S. 8). Erneut wird für ein direktes, markiertes Zitat Schlicht/Marsch als Quelle angegeben. Eine Recherche von Textauszügen aus der Seminararbeit führt zu dem Portal „Lehrerinnenfortbildung Baden-Württemberg“ ( $<$ https://lehrerfortbildung-bw.de $>$ ), einer nicht im Literaturverzeichnis angeführten Quelle, für die wiederum auf der Materialseite zu Julia Francks Kurzgeschichte kein Urheber genannt wird. Erst am Ende der Seite wird auf einen Beitrag Clemens Kammlers zu literarischen Kompetenzen verwiesen: „Kammler, siehe Rösch (Hrsg.) 2010: 197 ff.“ Unklar bleibt bei dieser Angabe, wer im Portal das Material erstellt hat und welche Teile dabei auf Clemens Kammler zurückgehen. Schlicht/Marsch finden dort jedoch keine Erwähnung.

In Beispiel 3 werden Schlicht/Marsch 2012 erneut ohne Angabe der Seitenzahlen zitiert. Der Text ist allerdings ebenfalls auf der Webseite der Lehrerinnenfortbildung auffindbar.

Die Online-Quelle wurde nicht in der Literaturliste aufgeführt. Der übernommene Text ist zwar zum Teil als Zitat markiert, jedoch erscheint es fraglich, ob die angegebene Quelle zu dem Zitat gehört. Eine Auseinandersetzung mit der Aussage aus der Quelle findet nicht statt, was auch durch das Latenthalten der Quellen und das Verschwimmen der Grenze zwischen Fremd- und Eigenmeinung unterbunden wird. Stattdessen kann man für Fall 3 und auch für Fall 4 feststellen, dass sich der Stil von Wikipedia, einen geschlossenen Text zu schreiben, bei dem Quellen bzw. Sachinformationen aus verschiedenen Quellen verarbeitet, zusammengefasst bzw. ergänzend aneinandergefügt werden, ohne dass expressis verbis auf die Herkunft der Quellen eingegangen wird, in den Seminararbeiten teilweise abgebildet findet. Diese Darbietung von Informationen unterscheidet sich also wesentlich von wissenschaftlichen Texten. In diesem Sinne können auch Texte von lehrerfortbildung- $b w$.de nicht als wissenschaftliche Texte angesehen werden, da auch hier die Aufbereitung und Weitergabe von Information Ziel der eingestellten Texte ist. Was also fehlt, ist eine Auseinandersetzung mit Quellen. Diese werden bestenfalls summarisch bei Wikipedia eingefügt (vgl. 
Wikipedia: Typologisches Modell der Erzählsituationen, a.a.O. Kritik an Stanzels Modell). Nicht die Autoren der Wiki-Artikel aber setzen sich mit den Inhalten auseinander, sondern sie geben lediglich die Auseinandersetzungen Dritter wieder.

Beide Fälle weisen neben den unmarkierten und „verschriebenen“ Zitaten noch ein weiteres Phänomen auf. Beide erwähnen das Eisberg-Prinzip als ein wesentliches Moment für die Erzählgestaltung bei Kurzgeschichten und die Interpretation der Geschichten (Fall 3, Seminararbeit S. 8 und 9. Fall 4, Seminararbeit S. 1, S. 2, S. 6). Zwar wird bei Fall 4 Folgendes hervorgehoben: „Der EisbergGrundsatz zufolge benötigt dem Autor ausschließlich den über das Meeresspiegel herausragende Teil zu beschreiben. Darunter steckende soll der Leser selbst aufzudecken“ (a. a. O., S. 6).

Aber in der Arbeit findet sich keine Anwendung des Grundsatzes für den Part des Lesers oder Forschenden, so dass weder der „herausragende Teil“ systematisch analysiert und „beschrieben“ noch der verborgene Teil aufgedeckt wird.

Fall 3 beschließt die Analyse der Kurzgeschichte Das Brot mit dem Hinweis auf das Eisberg-Prinzip. Eine Quelle zu der Information wird nicht genannt. Eine stringente Anwendung ist auch hier zu vermissen, obgleich es bei dieser Arbeit gelingt, in der Interpretation hinter den Verhaltensweisen der Figuren eine Gedanken- und Gefühlswelt aufzudecken und insofern die Parallelen zu dem auf Freud und Breuer zurückgehenden Eisberg-Modell für die Interpretation in gewisser Weise fruchtbar zu machen (vgl. <https://de.wikipedia.org/wiki/Eisbergmodell_(Literatur)>; 01.03.2019 sowie <https://de.wikipedia.org/wiki/Eisbergmodell>; 01.03.2019), wenn auch nur wenig Stringenz und Kohärenz zwischen der Erzählanalyse und der Interpretation bestehen.

Wie oben bereits erwähnt, ist das Latenthalten der verwendeten Quellen durchaus als ein Hindernis für die Auseinandersetzung mit den Quellen anzusehen. Daher gelingt es auch kaum, auf den aus Wikipedia adaptierten „Analysen“ eine sinnvolle Stringenz in der Interpretation herzustellen. Wikipedia dient als Stichwort-Lieferant. Zu einer vertiefenden Beschäftigung mit den Inhalten sowie einer fundierten Übertragung auf eine literaturwissenschaftliche Anwendung der Informationen gelangen die Studierenden jedoch kaum. Es lässt sich folglich nach der Intention der Quellenwahl und dem Nutzen der zitierten Quellen fragen.

\section{Fall 5: Markierte und unmarkierte Zitate als Hauptbestandteil der Interpretation}

Kommen wir nun zu dem fünften Fall. Herausgegriffen werden soll die Nutzung und Verwendung einer Quelle von grin.com (<http://www.grin.com/de/e-book/ 
275636/literarisches-lernen-sprachsensibel-anleiten-julia-francks-kurzgeschichte $>)^{2}$. Auch hier werden Teile aus der Quelle mit nur geringfügiger Variation unmarkiert übernommen, gerahmt von zwei direkten Zitaten. In gewisser Weise liegt also wiederum das Phänomen des „Verschreibens“ vor, bei dem Inhalte mehr oder minder intensiv paraphrasiert werden, jedoch dem Gehalt nach bei Kenntnis der Quelle dieser noch eindeutig zuzuordnen sind, ohne dass die Quelle für diesen Teil des Textes genannt wird. Ein anderes Phänomen ist jedoch bei dieser Arbeit und dem untenstehenden Auszug weit auffälliger. Unter der Rubrik „Interpretation“ erscheint ein 13-zeiliger Text zur Kurzgeschichte Streuselschnecke (Julia Franck 2002/2011), von dem knapp vier Zeilen ausgewiesene Zitate und weitere drei Zeilen unausgewiesene, indirekte Zitate sind. Das heißt, mehr als die Hälfte der als Interpretation bezeichneten Passagen in der Seminararbeit sind Fremdtext, so dass die eigenen Ausführungen sich auf sechs Zeilen beschränken. Eine Rückbindung an die Ausführungen zur Erzählanalyse unterbleibt vollständig. Die Inhalte der Zitate passen zudem keineswegs in eine Interpretation. Beide Zitate finden sich bei der angegebenen grin.com-Adresse in dem frei zugänglichen Textteil. Der zugehörige Forschungsartikel wurde nicht vollständig rezipiert.

Das erste Zitat befasst sich mit der Eignung der Kurzgeschichte für literarisches Lernen aufgrund der sprachlichen Gestaltung der Geschichte. Das zweite Zitat benennt die in der Kurzgeschichte auftretenden Konjunktivformen als Lerngegenstand. Während aber der Originaltext von Thérèse Remus, auch die Autorin des Textes wird in der Seminararbeit nicht erwähnt, im weiteren Verlauf darauf hinweist, dass der Konjunktiv nicht nur als ein grammatisches Phänomen im Kontext der Geschichte wahrgenommen werden sollte, sondern gerade auch seine „Funktion [...] vor allem mit Blick auf literarisches Schreiben“ sowie sein Einfluss auf „den Modus der Erzählung“ zum Lerngegenstand erhoben werden sollte (Remus 2013), entfällt dieser Aspekt in der Seminararbeit. Und so wird auch die von der zitierten Quelle ausgehende Anregung für die Auseinandersetzung mit dem Text nicht aufgegriffen. Eine eigene Vertiefung der Betrachtung von Gestaltung und Inhalt der Kurzgeschichte bleibt aus. Die „Interpretation“ endet mit dem Konjunktiv-Zitat.

Der große Anteil der markiert und unmarkiert verwendeten Zitate führt dazu, dass diese geradezu als Ersatz für einen eigenen Text erscheinen. Ferner sind beide Zitate inhaltlich zumindest in ihrer Handhabung nicht adäquat im Kontext einer Interpretation. Weder die Frage nach der Eignung der Geschichte für literarisches Lernen noch die der Geschichte zugeschriebenen geringen sprachlichen

2 Es handelt sich hierbei um eine Seminararbeit aus dem Jahr 2013, erstellt von Thérèse Remus an der Humboldt-Universität zu Berlin (Institut für Germanistik), Seminar: DaZ-Didaktik, 19 Seiten. 
Hürden (Zitat 1) lassen sich in irgendeinen sinnvollen Zusammenhang der Interpretation der Kurzgeschichte stellen. Das zweite Zitat hätte, wie aufgezeigt, das Potential, eine Interpretation der Kurzgeschichte partiell aufgrund der sprachlichen Auffälligkeiten anzuleiten, jedoch geschieht dies nicht. Die Verwendung des Zitates erscheint somit als deplatziert.

Für die zweite Kurzgeschichte, Das Brot, wird in der Interpretation eine chinesisch-sprachige Quelle ${ }^{3}$ verwendet, die als übersetztes Zitat in der Arbeit erscheint und ausgewiesen wird. Auch hier ist die quantitative Relation zwischen markiert-zitiertem Text und eigenem Text sehr unausgewogen. Von den 19 Zeilen sind 8 Zeilen ausgewiesenes Zitat. An einigen Stellen bleibt unklar, woher der Inhalt stammt. Gegenstand ist auch hier zum einen der Sprachstil der Geschichte, zum anderen aber auch das Licht-Motiv in der Geschichte.

Der Text schließt mit einem dreizeiligen Zitat, das nicht weiter erörtert und durch eigene Beobachtungen an der Kurzgeschichte diskutiert wird. Das heißt, auch für diesen Teil der Interpretation tritt zitierter Text an die Stelle einer eigenständigen Interpretation.

In ähnlicher Weise wurde bei Fall 1 und Fall 2 verfahren: Im ersten Fall wurden Versatzstücke aus der Internetquelle www.lerntippsammlung.de gleichsam von der ersten bis zur letzten Seite eingesetzt und durch „Verschreiben“ in den Text eingefügt. Zumeist wird am Ende einzelner Absätze auf die Quelle verwiesen, ohne dass jedoch in dem Text selbst eine Abgrenzung zwischen Fremd- und Eigenmeinung erfolgen würde. Auch werden einzelne Aspekte der Aussagen der Quelle nicht weiter erörtert oder durch eigene Beobachtungen am Primärtext ergänzt und kommentiert.

Im zweiten Fall wurden Zitate zwar durchaus markiert, aber oftmals vergleichsweise unpräzise wie in Fall 1. Zudem werden bereits im Einleitungstext und auch weiter fortlaufend Bewertungen der Novelle Tod in Venedig von Thomas Mann (1992 [Erstdruck 1913]) sowie ihrer Erzählgestaltung vorgenommen, die in der Seminararbeit selbst nicht entwickelt werden. Auch werden keine Quellen der Erkenntnisse genannt, vielmehr wird das Ausgesagte als Gegebenheit hingestellt. Es entsteht dabei der Eindruck der Übernahme aus der Sekundärliteratur, die als unmarkiert referiert erscheint.

Die eigene Leistung, das heißt eine eigenständige Analyse, wird auch nicht der Darstellung in der Internetquelle gegenübergestellt, Quellen werden nicht als Fußnoten mit konkreten Stellen im eigenen Text verbunden, sondern erscheinen meist summarisch am Schluss eines Teilkapitels oder lediglich in der Literaturliste. Das heißt, nicht nur werden Fremdleistung und Eigenleistung verschmolzen, sondern

3 Feng (1986). 
es wird damit auch eine explizite Auseinandersetzung mit und Erörterung von Quellen - ein fundamentaler Aspekt wissenschaftlichen Arbeitens - unmöglich.

An einem Wissen um die Bedeutung der Bearbeitung von Quellen sowie der kritischen Auseinandersetzung mit Quellenmaterial und eigenen Untersuchungen als essentiell für wissenschaftliches Arbeiten scheint es mithin noch zu mangeln, so dass dieser Punkt ausdrücklich thematisiert werden muss.

\section{Wissenspartizipation - Wissensvermittlung und Wissenschaftsrezeption}

\subsection{Relevanz von Arbeitsweisen im Kontext der Globalisierung - Wissenschaftstheoretische Perspektive bei Neuser}

Mit dem Fortschreiten der Globalisierung und dem Ausbau der Internetpräsenz wissenschaftlichen Wissens gewinnt das Erlernen internationaler Standards in den Wissenschaften an Bedeutung. Den Konsequenzen und Herausforderungen für einzelne Wissen(schaft)skulturen widmet sich Neuser, indem er eine globale Weltwissensgesellschaft als ein im Entstehen begriffenes Szenario darstellt (vgl. Neuser 2013: 5). Dabei stuft Neuser zum einen die Bedeutung des Wissens und die Verfahren der Wissensgenerierung und zum anderen auch die Möglichkeiten und die Bereitschaft zur Partizipation an dem globalen Wissenssystem innerhalb unterschiedlicher Gesellschaften als essentiell für das jeweilige gesellschaftliche Vermögen ein, per Partizipation am Wissen selbst sowie an den damit verbundenen Erfahrungsräumen teilzuhaben.

Die sich neu konzipierende Weltwissensgemeinschaft schafft sich darin ihren eigenen Erfahrungs- und Handlungsraum, aus dem heraus sich weiteres Wissen generiert. Wissen ist weiterhin von dem „Kulturkreis“ (Neuser 2013: 10), in dem es entsteht, abhängig, jedoch weitet sich der „Kulturkreis“, indem ein global vernetzter Erfahrungsraum kreiert wird (vgl. Neuser 2013: $45 \mathrm{ff}$.).

Auch der virtuelle Austausch führt $\mathrm{zu}$ einem Zusammentreffen von lokalen Kulturen im globalen Raum. Die daraus resultierende Rezeption von Wissen bedeutet nach Neuser eine Herausforderung für die rezipierenden Kulturen (vgl. Neuser 2013: 314).

Neuser merkt an, dass die konkrete Wissensrezeption entweder durch eine Übernahme von fremden Topoi oder durch deren Neudeutung vor dem je eigenen Hintergrund der rezipierenden Kultur ein Phänomen der sich globalisierenden Wissensgesellschaft sei. Dabei bezeichnet Neuser die Neudeutung als „Fälschung“ (vgl. Neuser 2013: 315). 


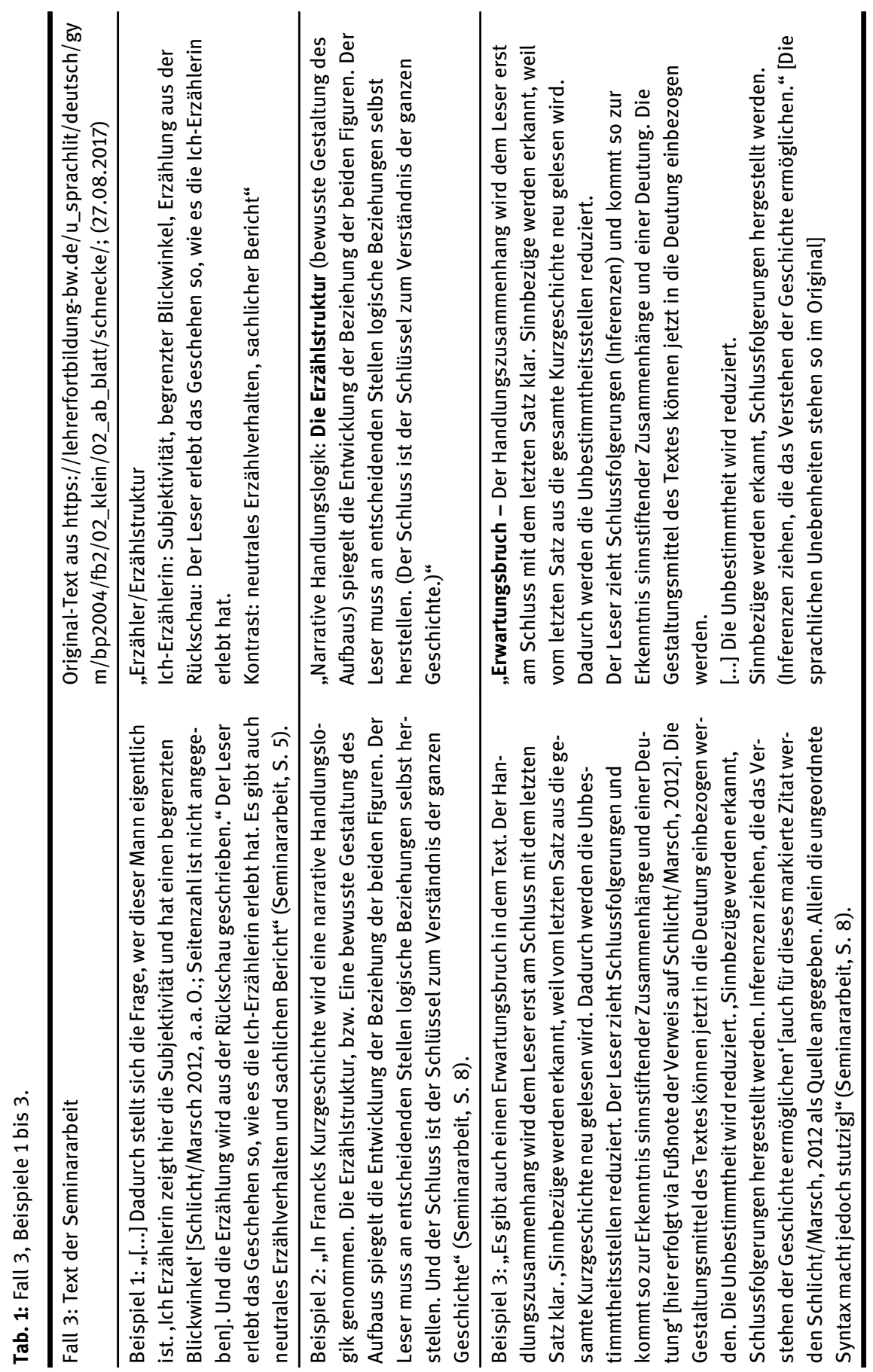


Ob eine rezipierende Kultur Begriffe übernehme oder aus dem eigenen Kontext neu deute, hängt nach Neuser davon ab, ob die Kulturen in ihren Entwicklungen historisch stark voneinander abgegrenzt waren, sei es geographisch, sei es aufgrund anderer Zugangsmöglichkeiten zu kulturellen und Erfahrungsräumen (vgl. Neuser 2013: 318ff.).

Kennzeichnend für die gegenwärtige Situation der Globalisierung ist der Umstand, dass gleichzeitig „verschiedene Kulturräume der globalisierten Welt stark“ (Neuser 2013: 322) aufeinander Einfluss nehmen.

Wir müssen aushalten, dass wir Wissen immer nur als Konstrukt einer Beziehung von Begriff, Erfahrung und Handlung präsent haben und unsere Konstruktion von Welt keine zeitlose Bedeutung hat, sondern an unsere Zeit und unsere Kultur gebunden ist. (Neuser 2013: 326)

Durch den multilateralen Austausch baut sich jenes Wissenskonzept auf, das „Wissen als ein dynamisches selbstorganisiertes System begreift“ und ,jenseits der Subjekte im Sinne der Neuzeit“ im Entstehen ist“ (Neuser 2013: 326). Dies führe dazu, dass aus globaler Perspektive nicht mehr ein „Zusammentreffen einzelner Kulturen [stattfinde], sondern vielmehr [die Wissenskultur] wie eine Kultur erscheine“ und agiere, denn da Wissen sich um Fragen herum entwickele, werde zukünftig eine neue „gemeinsame [globale] Fragestellung [...] entwickelt“ (Neuser 2013: 326f.).

Der Präsenz dieser „Vorstellung von Wissen“ schreibt Neuser weltweite Gültigkeit zu und sieht sie als Basis jener sich herausbildenden „neuen Kultur der globalisierten Wissensgesellschaft“ (Neuser 2013: 330).

Allerdings zeigen Untersuchungen zur ökonomischen Bedeutung von Wissen, dass der Prozess der Herausbildung einer global-globalisierten Wissensgesellschaft bis dato aufgrund fehlender sozialer und kultureller Prozesse etwa in einigen Regionen Südostasiens erst an seinen Anfängen steht (vgl. Evers et al. 2004: 6). Eine Einbindung jenes Wissens aus Gesellschaften, die noch nicht oder nur wenig an der Entwicklung der Weltwissensgesellschaft partizipieren, erscheint jedoch aus Sicht einer Inklusion verfügbaren Wissens in jenes System der entstehenden Weltwissensgesellschaft erstrebenswert (vgl. Otto 2018: 107).

Bei der Integration verschiedener „Kulturkreise“ darf nicht vernachlässigt werden, dass die sich mit ihrem Wissen integrierende Gesellschaft in der Partizipation einen echten Gewinn für sich sehen muss (vgl. Schiewer 2010: 137). Wenn etwa ein Wissen über die Lern- und Wissen(schaft)skultur in das globale System eingegeben wird, muss auch die gebende Gesellschaft an Entscheidungen über die Prozesse der weiteren Generierung von Wissen beteiligt sein, soweit dies systematisch möglich ist, allein schon damit gewonnenes Wissen anschließbar bleibt und in die je eigene Kultur zurückwirken kann. 
Das insgesamt im Prozess der Globalisierung sich entwickelnde gemeinsame Wissen schafft wiederum die Basis für eine Integration von Wissen. Neue Formen auch der Zusammenarbeit sowie möglicherweise auch des Erkenntnisgewinns in den Wissenschaften wären eine etwaige Folge. Darin liegt auch der Nutzen eines Austausches über Lernkulturen.

\subsection{Erkenntnisse der Transferwissenschaften - Aktive Partizipation in Transfer und Austausch}

Den Akteuren und Aktionen im Prozess des Wissensaustausches oder Wissenstransfers widmen sich die Transferwissenschaften mit dem Ziel, Wege der Zirkulation und Generierung von Wissen zu ermitteln, um so „sprachliche[] bzw. mediale[] Formen von adressatenspezifischen Lösungen für gelingende Rezeption von bestimmten Informationskomplexen und Wissensbeständen“ (Antos 2013: 226) bereitstellen zu können.

Eine Partizipation am digital verfügbaren Wissen bedeutet zunächst eine deutliche Zunahme eines unvermittelt, autodidaktisch erwerbbaren Wissens als spezifische Konstellation zwischen Wissensgeber und Wissensnehmer, die den Wissenstransfer bestimmt. Antos merkt hierzu an, dass ein Wissen, welches von seinem Entstehungshintergrund entkoppelt ist, zur Information wird, die in der Rezeption erst erneut als Wissen „revitalisiert“ werden muss (vgl. Antos 2005: 358 sowie Antos 2013: 226). Die damit verbundene Abkehr von einer didaktisierten Wissensvermittlung verschafft der konstruktivistischen Sichtweise im Rezeptionsprozess ein stärkeres Gewicht, lässt sich mit Dewe und seinem Verweis auf Kroner und Wolff vermerken (vgl. Dewe 2005: 370ff.; Kroner/Wolff 1984: 453). Sprechen Kroner/Wolff (ebd.) von einer „Herstellung von Ähnlichkeit zwischen Wissenschaft und einem besonderen Praxisbereich“, so beschreibt Dewe den Transferprozess dabei als „Adaptation“ (Dewe 2005: 371), bei der die Wissensentnahme „als kluge Applikation wissenschaftlichen Wissens nach spezifischen Situationen“ des Rezipierenden erfolgt, um durch das Wissen zu „praktischen Problemlösungen“ zu gelangen (ebd.). Für den Rezipienten geht es dabei nicht darum, ein Wissen möglichst vollständig zu erwerben. Vielmehr entnimmt er Wissen für seinen Bedarf einer Problemlösung.

Verlagert man die Erkenntnisse aus den Transferwissenschaften auf eine Metaebene, so lassen sich Dewes theoretische, auf den Wissenstransfer zwischen Theorie und Praxis gerichtete Überlegungen auf die Lernprozesse und die Verarbeitung von Wissen in Seminararbeiten übertragen und die dortigen Verfahrensweisen mit dem Grundmuster der „Applikation“ assoziieren. Geht es doch den Studierenden auf der praktischen Ebene darum, das geforderte Seminar- 
papier zu erstellen, wofür sie vorhandenes Wissen den Quellen entnehmen und es in ihre Seminararbeit einfügen bzw. es zur Erstellung ihres Seminarpapieres benutzen, womit ihr Schreibproblem gelöst ist.

Des Weiteren beschreibt Dewe das Verhältnis zwischen Theorie und Praxis als komplementär. Beide Seiten beobachten sich gegenseitig. Im Rückgriff auf Luhmann bemerkt Dewe (2005: 371; vgl. Luhmann 1987: $36 \mathrm{ff}$.), dass sich über die Beobachtung ein eigener Wissensbereich herausbildet.

Dieses gegenseitige Beobachten erscheint als ein wesentliches Moment im Kontext des globalen Wissensaustausches. Es ist auf die Inhaltsseite gerichtet. Dabei steht das Wissen als Information im Mittelpunkt und zwischen der jeweiligen wissensgebenden Seite mit ihren zugehörigen kulturellen Deutungsmustern des Wissens und der wissensnehmenden Seite mit eigenen, auf kulturellen Prägungen basierenden Deutungsmustern sind Aushandlungsprozesse über die Bedeutung der Information erforderlich. Dabei muss es nicht zwingend zu einem Konsens kommen (vgl. Schiewer 2010: 136f.). Relevanz gewinnt die Kenntnis der Deutungs- und Verstehenshorizonte der jeweils anderen Seite dadurch, dass Bedingtheiten des Verständnisses bewusstgemacht werden und wissenschaftliches Wissen auch fortgeschrieben werden kann.

Loenhoff hält zur Theorie-Praxis-Relation fest, dass aus wissenschaftlichem Wissen praktisches Wissen nicht erschlossen, sondern nur in der Praxis erfahren werden kann (vgl. Loenhoff 2014: 26). Dies resultiert unter anderem daraus, dass Praxis über eine Flexibilität verfügt, die für Theorie mit der begrifflichen Fixierung von Wissen gerade nicht charakteristisch ist (vgl. Dewe 2005: 369ff.). Prozessuales sowie implizites Wissen können also nur in der Begegnung erworben werden (vgl. Loenhoff 2014: 26ff.). Dieser Umstand ist ein wichtiges Moment nicht nur in der Rezeption wissenschaftlichen Wissens, sondern auch in der Aneignung von Formen wissenschaftlichen Arbeitens, die aus differenten Lehr-Lern-Situationen resultieren.

Die Bewusstheit gegenüber den regionalen Implikaturen im Wissen stellt einen Punkt in der Adaptation von Wissen dar, dem systematisch größere Aufmerksamkeit geschenkt werden muss. Es erscheint als ein zentraler Punkt in der Kommunikation von Wissen, dass eine Kommunikation über Wissen und seine Bedingtheiten zum einen präsent sein und dass diese Bedingtheiten zum anderen auch konkret nachvollziehbar bleiben müssen. 


\subsection{Fazit Wissenschaftspartizipation - ein Vergleich von wissenschaftstheoretischer und transferwissenschaftlichen Perspektiven}

Verbindet man die Ausführungen von Neuser zur Entwicklung einer globalen Wissensgesellschaft mit den vielfältigen Untersuchungen zum Transfer von Wissen, dann lassen sich zunächst zwei Punkte festhalten, in denen sich beide Forschungsterrains überschneiden. Sowohl Neuser als auch die Transferwissenschaften gehen von einem Wissensgefälle als Input für Wissenstransfer bzw. Bemühungen um Partizipation am Wissen aus (vgl. Neuser 2013: 314; Wichter 2005: 411). Ferner ist es die Feststellung, dass die Generierung von Wissen personell und räumlich-zeitlich verankert ist (ebd.). Während aber Neuser die potentiell eher Wissen nehmende Seite gleichsam in der Pflicht sieht, Anschluss an heutige Prozesse der Wissensgenerierung und -dissemination zu finden (vgl. Neuser 2013: 331f.), fokussieren die Transferwissenschaften ein breites Spektrum an Konstituenten, die am Prozess der Dissemination beteiligt sind (vgl. unter anderen: Wichter 2005: 409ff.; Dewe 2005: 368ff.; Ballod 2004: 105ff.).

Für Neuser stellt die Beteiligung am globalen Wissensaustausch zugleich die Teilhabe an dem sich damit neu konzipierenden Erfahrungs- und Handlungsraum dar, der nach eigenen Regeln auch neues Wissen generiert (vgl. Neuser 2013: 330). Es lässt sich anfügen, dass eine Partizipation jedoch nicht nur ein Nutzen vorhandenen Wissens bedeutet, sondern die Verwendung neuen Wissens führt zu einer Verarbeitung von Wissen, so dass wiederum weiteres Wissen Eingang ins System findet. Zwar gewinnt das globale System auch eine Eigendynamik, so bleibt es zugleich aber regional und lokal individuell verankert. Partizipation hat eine Diversifizierung des Wissens zur Folge und verlangt danach, sich unterschiedlicher lokaler Einflüsse bewusst zu sein.

Diese Praxis, lokale Bedingtheiten ebenfalls in das System verstärkt einzuspeisen, scheint ein Desiderat zu sein, um explizites Wissen möglichst adäquat erschließen zu können. Diskutierenswert sind dazu weitere technische Möglichkeiten des Internets, die es erlauben, auch Einblicke in Handlungswissen und lokale Praktiken zu gewähren, um zum einen eine internationale Anschließbarkeit weiterhin zu garantieren. Zum anderen wird dies insbesondere dort wichtig, wo es nicht einzig darum geht, ein Wissen in einen eigenen neuen Kontext zu integrieren, sondern auch darum, ein Wissen verfügbar zu haben für eine weitere Vermittlung an Lernende, die sich etwa auf einen Aufenthalt in der Zielkultur der Fremdsprache vorbereiten. Aufmerksamkeit ist eine wichtige Voraussetzung für das Verständnis, jedoch reicht sie nicht zwingend aus, um regionalspezifische Bedeutungen eigenständig aktivieren zu können. Hier zeigt sich erneut der Bedarf an Kontextualisierung von Wissen bzw. von Information auch im Netz. 


\subsection{Kontextualisierung - Gegenstand der Kulturwissenschaften}

In seiner kulturtheoretisch fundierten Landeskunde, in der es um die Kontextualisierung von „Texten“ geht, setzt Altmayer sich detailliert mit dem Begriff „Kultur" auseinander und intendiert damit eine Abkehr von einem nach Ethnien oder Nationen homogenisierenden Kulturbegriff (vgl. Altmayer 2004: 112ff.). Die Diskussion des Terminus „Kultur“ schließt dabei an die Ausführungen bei Welsch an (vgl. Welsch 1997: 67ff.). Danach ist „Kultur“ nicht durch subjektunabhängige Begriffe präfiguriert, sondern kulturelle Identität entwickelt sich subjektiv. Zwar finden einerseits auch Prägungen des individuellen Verständnisses durch Größen wie Ethnien etc. Eingang in die kulturelle Identität, andererseits aber stehen den eher zum Monolithischen tendierenden Einflüssen zugleich allein durch die Globalisierung und den internationalen Austausch diverse Einflüsse gegenüber. In der Summe gestattet dies eine individuelle kulturelle Bestimmung (vgl. Welsch 1997: 78ff.). Dabei kommt es allerdings zu partiellen Überschneidungen bzw. Vernetzungen mit anderen Individuen. Selbstverständlich wird aber „Kultur“ nicht nur auf der Mikroebene festgelegt, vielmehr definiert sich „Kultur“ auch für die Makroebene (vgl. Welsch 1997: 71ff.). Hier ist es nicht mehr eine vorab fixierte Bestimmung von „Kultur“ mit weitreichender Gültigkeit für die Mitglieder einer Gesellschaft, sondern kulturelles Selbstverständnis wird auch hier von der Erfahrungsgemeinschaft immer wieder neu ausgehandelt und bestimmt (vgl. Welsch 1997: 73ff.).

Statt Zuschreibungen an den „Text“ wird dieser über die „Kommunikationsgemeinschaft“ durch weitere „Texte“ kontextualisiert und darüber in seinen kulturellen Deutungsmustern erschließbar. Ziel ist es nicht, einen eindeutigen Sinn zu ermitteln, vielmehr dienen die Kontexte dazu, den Ausgangstext in seinen Bedeutungen zu erhellen und die Präsuppositionen sichtbar zu machen (vgl. Altmayer 2004: 244f.).

Mit diesem sensiblen, von Altmayer als „Hypertext“ bezeichneten Verfahren, bei dem es weniger um Festlegungen einer Deutung als vielmehr um bestenfalls eine Art Rahmung von Wissen bzw. mehr noch um ein Erhellen und Beleuchten eines „Textes“ geht , ist insbesondere angestrebt, durch vielfältige Bezüge von Stereotypisierungen Abstand zu nehmen, um eine Abkapselung von Sinnbezügen zu hintergehen und den „Text“ auf seine (Be-)Deutungsmuster hin zu öffnen (vgl. Altmayer 2004: 255ff.). Neben dem Entstehungskontext ist aber auch eine Art Parallelkontext zum lebensweltlichen Hintergrundwissen (vgl. Altmayer 2004: 146) des Rezipienten zu eröffnen, so dass dieser neues Wissen integrieren kann. Daraus folgt für eine im Kontext des Faches DaF ausgelegte kulturwissenschaftliche Landeskunde, dass eine Kontextualisierung zur Erweiterung des Ausgangstextes und 
des Einbeziehens von lebensweltlichem Hintergrundwissen für eine Beteiligung nicht-muttersprachlicher Sprecher^innen des Deutschen offen ist, die an der Kommunikationsgemeinschaft teilhaben.

Eine Deutungshoheit, die ohnehin nicht unmittelbar auf den Text selbst ausgerichtet ist, sondern eben lediglich die Ebene des Impliziten und Präsupponierten des Ausgangstextes erhellen möchte, liegt nicht in einer homogenen Gruppe und ist auch nicht monokulturell ausgerichtet, sondern sie eröffnet Wege, auch Texte multiperspektivisch zu erschließen und so auch die Mehrbezüglichkeit des Ausgangstextes zu berücksichtigen.

Dass eine transkulturelle Vernetzung als Basis für den Wissensaustausch ertragreich ist, zeigen Li Shuangzhis Untersuchungen zur Rezeption von Xi Chuans zivilisationskritischem Prosagedicht Schmetterlinge auf der Autobahn im deutschen Feuilleton (vgl. Li 2009: $141 \mathrm{ff}$.). Das Misreading auch verschiedener weiterer Gedichte resultierte daraus, dass das im deutschen Kontext vertraute Deutungsmuster der Zivilisationskritik ohne genauere Kenntnisse der spezifischen chinesischen Bezüge zum „Schmetterlingsmotiv“ auf die chinesische Lyrik angewendet worden war. Die Kontextualisierung durch Li Shuangzhi offenbarte als Kontext einen im Daoismus wurzelnden Text des Dichters Zhuangzis vom Schmetterlingstraum. Diese Situierung des Textes eröffnet ein erweitertes Rezeptionsspektrum und erlaubt auch fremdsprachigen Rezipierenden, den in China begonnenen Wandel der Modernisierung in seiner Tragweite für das Selbstbild und Selbstverständnis, aber auch das Verhältnis von Mensch und Natur tiefer zu verstehen.

Für die Kontextualisierung ist ein Austausch zwischen verschiedenen Erfahrungsgemeinschaften ertragreich, um unterschiedliche Präsuppositionen aufdecken, die erforderlichen kulturellen Deutungsmuster aufrufen und eine adäquate Interpretation des Ausgangstextes leisten zu können. Zudem lässt sich aus der Perspektive der Transkulturalität festhalten, dass in dem Moment der Zivilisationskritik ein globales Problem der Gegenwart angesprochen ist, während mit der Konkretion des Phänomens ein regional-spezifisches Moment eines kulturellen Deutungsmusters hinzutritt (vgl. Welsch 1997: 79f.). Diese Doppelheit von „global“ und „partikular“, wie Welsch (vgl. 1997: 80) es nennt, ist charakteristisch für transkulturelle Identitäten.

Zusammenfassend lässt sich sagen, dass Teilhabe am Wissen in einem wesentlichen Maße durch Kommunikation und Interaktion in Bezug auf das Wissen geleitet wird. Ein weiteres wesentliches Merkmal ist die Erweiterung des Rezeptionsgegenstandes durch ergänzende und erhellende Informationen, die dem Aushandeln von Bedeutungen dienen. 


\subsection{Studentische Arbeitsweisen in transfer- und kulturwissenschaftlicher Perspektive}

Betrachtet man das Zitationsverhalten noch einmal vor dem Hintergrund der Darlegungen des Wissenstransfers und der Ausführungen zur Weltwissensgesellschaft, so lässt sich festhalten, dass hinsichtlich der Praktiken wissenschaftlichen Arbeitens und des entsprechenden Wissens dazu durchaus noch einige „Rezeptionswiderstände“ (vgl. Dewe 2005: 370ff.) bei der Wissensaufnahme bestehen. Wenn auch die Studierenden im Seminargeschehen in großem Umfang mündlich sowohl eigenständig Texte analysierten, Aussagen aus Quellenmaterialien mit eigenen Positionen verglichen, so findet sich diese Praxis in den Seminararbeiten nicht eindeutig wieder. Das Verfahren der Kombination von markierten und unmarkierten Zitaten zur Erstellung eines Textes ist mit dem Vokabular der Transferwissenschaften dahingehend zu bewerten, dass diese partielle „Adaptation“ von Wissen (vgl. Dewe 2005: 371) keine angemessene Partizipation (vgl. Dewe 2005: 370f.) am wissenschaftlichen Diskurs erlaubt. Zwar bewirkt die spezifische Art der Zitatverwendung ein Problemlösen auf der „Verwenderseite“ (ebd.), nämlich einen Text zu schreiben, jedoch bedeutet das Verfahren der Nicht-Markierung eine gewisse „Zuschreibung“ hinsichtlich der Verwendungsmöglichkeiten von Quellen. Mit obiger Terminologie lässt sich sagen, dass die „kulturellen Deutungsmuster“ (vgl. Altmayer: 154) für die Funktion des Schreibens eines Textes im akademischen Kontext sowie die Markierung von Zitaten offenbar nicht einsichtig geworden sind und mit Neuser (2013: 315) als „Fälschung“ verstanden werden können.

Die (kulturelle) Praxis, markierte und unmarkierte Zitate anstelle eines eigenen Textes zu setzen, wirft die Frage nach Handlungsgewohnheiten und vertrauten Erwartungsmustern im Umgang mit Wissen, Wissensaneignung und Wissensproduktion auf. Da es sich beim wissenschaftlichen Arbeiten auch um ein Handlungswissen handelt, von dem es heißt, dass es nicht erschlossen, sondern nur erfahren werden kann, ist zu fragen, welche Kontextualisierungen (vgl. Altmayer 2004: 151ff.) erforderlich sind und wie sie herzustellen sind, damit eine angemessene Partizipation am wissenschaftlichen Diskurs ermöglicht wird.

Das „kulturelle Deutungsmuster“ (vgl. Altmayer 2004: 154) der Positionierung von Forschungsmeinungen und ihrer Diskussion durch Positionierungen weiterer Forschender mit Bezugnahme auf vorhandene Forschung müsste also kontextualisiert und als Verfahren anschaulich gemacht werden. Dazu wären Prozesse des Austausches und auch der Aushandlung zwischen Wissenschaftler*innen der beteiligten Lern- und Wissenschaftskulturen nützlich, so dass ein umfassendes Wissen hinsichtlich des vielschichtigen impliziten und prozessualen Wissens, das jeweils in den Kulturen eingeschlossen ist, transparent gemacht werden könnte. 
Die Positionierung gegenüber Quellen, die Reaktion auf verwendete Quellen sowie das Präsenthalten der ursprünglichen Bedeutung eines Quelltextes und die Einordnung des Wissens in den wissenschaftlichen Diskurs und möglicherweise auch die gesellschaftliche Rahmung als Handlungsfeld, in dem jeder neue wissenschaftliche Text agiert, sind dabei wesentliche Aspekte.

Diese Kontextualisierung einsichtig zu machen, wird jedoch durch die Internetpräsenz eindeutig erschwert. Die Omnipräsenz leistet einer Entkontextualisierung von Wissen Vorschub. Das ist gerade auch bei den niedrigschwelligen Texten von Wissen, wie sie in Seminararbeiten verwendet werden, zu beobachten.

\section{Internetformate - Plattformen für Austausch, Interaktion und Kontextualisierung - Möglichkeiten und Desiderate}

Im Folgenden sollen einige Formate aus der Germanistik vorgestellt werden, die im Hinblick auf Aushandlungen von Bedeutungen und Deutungsmustern bereits aktiv waren und geeignet erscheinen, ein Spektrum an Texten im Sinne Altmayers mit einem „lebensweltlichen Hintergrundwissen“ zu „kulturellen Deutungsmustern“ (vgl. Altmayer 2004: 142f.) aufzunehmen und auch interaktive Aushandlungen von (Be-)Deutungen zu gestatten. Ziele dieser anvisierten Interaktionen sind die Partizipation an und Dissemination von Wissen etablierter Wissenschaftler^innen auf dem Gebiet des Fachwissens, so dass auch Jung-Akademiker^innen aus dem nicht-muttersprachlichen Kontext Wege zur Anschließbarkeit von Wissen aus der Fremdsprachenphilologie finden können. Darüber hinaus gilt es, ein Handlungswissen zum wissenschaftlichen Arbeiten und dem wissenschaftlichen Fachdiskurs für Nachwuchswissenschaftler`innen einsehbar zu machen. Nicht zuletzt kann dieses Wissen für Akademiker*innen aus anderen Disziplinen nützlich sein.

Anhand von fünf Beispielen werden Möglichkeiten aufgezeigt, die das Internet bietet, um Formen der Wissensvermittlung und Wissenspartizipation über eine bloße Informationsentnahme hinaus zu ermöglichen.

Auf Seiten der Wissensgeber sind es etablierte Wissenschaftler*innen, insbesondere der Fremdsprachenphilologien, die sich individuell bereits Wege zu fremdkulturellem Wissen erschlossen und oftmals auch die Lern- und Wissenskulturen kennengelernt haben und dadurch mit zwei Lern- und Wissenschaftskulturen vertraut sind. Dieses individuell verfügbare Wissen fließt zumeist lediglich implizit in die weitere fachliche Wissensvermittlung ein, wird aber, anders als das erworbene Fachwissen an sich, in vergleichsweise geringem Umfang explizit 
gemacht. Hier liegt ein Potential, dieses Wissen ebenfalls zu erschließen und interessierten Wissensnehmenden im Netz zugänglich zu machen. Diese Erweiterung des Fachwissens kann ganz im Sinne von Altmayers Wissen um kulturelle Deutungsmuster verstanden werden.

Die Kulturwissenschaft [...] stellt vielmehr selbst Bezüge zwischen Texten her, die weder ,wahr' noch ,falsch', sondern lediglich ,sinnvoll‘ oder ,nicht sinnvoll‘ im Hinblick auf fremdkulturelle Lern- und Verstehensprozesse sein können. (Altmayer 2004: 265)

\subsection{Das Projekt IDIAL - Formate für Fachdiskurse - Leichte Sprache}

Als Erstes sei das Projekt IDIAL (vgl. Middeke 2009: 45ff.) genannt. Gemeinsam haben Slawisten und Germanisten für die Slawistik in Göttingen und verschiedene Germanistische Institute in Ländern mit slawischen Sprachen Lehrwerke entwickelt, die sowohl den Lerntraditionen der Studierenden als auch den Zielkulturen gerecht wurden. Für die Studierenden ist somit die Möglichkeit des kulturellen Lernens gegeben. Im Verlauf der Ausarbeitungsprozesse der Lehrwerke hat ein Austausch auch über Bedeutungen verschiedener Themenfelder stattgefunden, der partiell auch in die Lehrwerke eingeflossen ist, zum Teil jedoch lediglich als implizites Wissen Eingang gefunden hat. Hier sehe ich einen Ansatzpunkt für weitere Forschungen, in denen genau diese Aushandlungsprozesse selbst zum Gegenstand werden.

Als Zweites ist die Annotierte Bibliographie zur Literaturtheorie der Göttinger Universität zu nennen, die ein Glossar integriert hat (vgl. www.literaturtheorie. uni-goettingen.de). Zugegeben, dieses Format ist noch nicht in „leichter Sprache“, aber es erlaubt jenseits von Wikipedia, sich fachliches Grundwissen anzueignen. Das heißt, eine Erweiterung um die Rubrik „Leichte Sprache“ wäre wünschenswert und auch vorstellbar.

Das dritte Projekt li-go.de (vgl. www.li-go.de) richtete sich ausdrücklich an Studierende der Literaturwissenschaften. Leider blieb es Fragment. Neben dem an studentische Bedürfnisse angepassten Wissens- und Sprachniveau bot die integrierte Forumsfunktion Studierenden Möglichkeiten zum Austausch über das Wissen. Derartige bi- und multikulturell betriebene Formate könnten zukünftig in unterschiedlichen Disziplinen sowohl einen Austausch über fachliche Informationen und ihre Bedeutungen als auch über Formen der Verarbeitung wissenschaftlichen Wissens ermöglichen.

Als viertes Beispiel soll aus dem Bereich Germanistik ein Online-Fachjournal angeführt werden: Textpraxis von der Universität Münster (vgl. www.uni-muenster.de/Textpraxis/en). Neben der Publikation von Fachartikeln ist eine Kommen- 
tar- sowie eine Forumsfunktion verfügbar, die Wissenschaftler^innen mit unterschiedlichem kulturellem Hintergrund Gelegenheit bietet, in einen fachlichen und zugleich kulturellen Austausch zu treten und damit auch Jung-Akademiker^innen zu einem adäquaten Verständnis der Texte zu verhelfen.

Als letztes ist die Organisation „Wissenschaft im Dialog“ (vgl. www.wissenschaft-im-dialog.de) zu erwähnen. Sie versammelt einerseits Online-Aktivitäten, andererseits versteht sich das Format als eine Plattform zur Präsentation von analogen Aktivitäten. Es zielt vorrangig im technisch-naturwissenschaftlichen Bereich auf einen Wissensaustausch zwischen Experten und Laien, damit eine breite Partizipation an neuem Wissen möglich wird und das in verschiedenen Wissenschaften gewonnene Wissen von einer breiteren Schicht je nach eigenen Interessen rezipiert werden kann. Entwickelt wurde auf diesem Weg ein umfassendes Spektrum an Formen der Wissenspräsentation, der Wissensvermittlung und auch der Wissensaneignung.

Im Herbst 2017 befasste sich das Forum Wissenschaftskommunikation explizit mit Fragen nach Kommunikationstechniken und Kommunikationswegen (vgl. ebd.). Dabei geht es um Wege der Vermittlung von Wissen sowie um Wege der Informationsrezeption. Neben den Akteuren spielen Fragen nach der Verwendung von Medien eine Rolle. In der Diskussion über barrierefreie Zugänge zu Wissen ist die Rubrik „Leichte Sprache“, wie sie als Format von Internetauftritten verschiedener Medienanstalten bereits zur Dissemination von Nachrichten Verwendung findet, ein wichtiger Unterpunkt. Die Aufbereitung von Wissen als Information in „Leichter Sprache“ erscheint als ein wichtiges Zugangstor zu dem in der Information auch implizierten Wissen.

Nicht zuletzt technisch bestehen bereits einige Formate, im Internet den Wissenserwerb zu begleiten und Optionen offenzuhalten, um plurikulturell in einen fachlichen Austausch über Lern- und Wissenschaftskulturen zu treten und auch im Austausch mit Jung-Akademiker*innen diesen den Einstieg in den internationalen Diskurs durch eine Moderation des expliziten, impliziten und auch prozessualen Wissens zu ebnen. Dies erlaubt Studierenden kulturelles Lernen, indem vertraute und weniger vertraute Perspektiven gemeinsam in Erscheinung treten und lokalspezifische Sichtweisen und Formen des Diskurses mit plurikulturellen und internationalen Gepflogenheiten abgeglichen werden können.

\subsection{Fazit - Wissenschaftlicher Austausch}

Insgesamt lässt sich festhalten, dass für die Vermittlung von Fachwissen ein stetiges Angebot der Moderation verfügbar sein müsste. Einerseits ist ein kontinuierlicher Bedarf an niedrigschwelligem Zugang zu wissenschaftlichem Wissen und 
dem darin enthaltenen Wissen über Wissensgenerierung in den Wissenschaften sowie Formen wissenschaftlichen Arbeitens mit einem Zusatzangebot, auch individuell Fragen zum Verständnis adressieren zu können, vorhanden, so dass entsprechende Angebote, die diesen Bedürfnissen entgegenkommen, mehr als wünschenswert sind.

Andererseits aber verlangt auch die High-End-Wissenschaft nach einer erweiterten Kommunikation, so dass Aushandlungen über Bedeutungen aus unterschiedlichen kulturell geprägten Perspektiven explizit gemacht werden können. Darin kann ein Beitrag geleistet werden, ein Grundverständnis von Texten zu erzielen und darin das Erschließen von Texten für Nachwuchswissenschaftler`innen anzuleiten. Darüber hinaus würden über die Präsenz der Aushandlungen von Semantiken und ihrer Prozesse im Internet Einblicke in regionale kulturelle Einflüsse gewährt, auch dort unabhängig davon, ob es zu einem Einverständnis kommt oder ein bewusstes Nebeneinander von Bedeutungen bestehen bleibt.

Kulturelle Deutungsmuster bezüglich der Kultur der jeweils wissensgebenden Seite wären mit kulturellen Deutungsmustern der wissensnehmenden Seite hinsichtlich Übereinstimmungen und auch Divergenzen erkennbar. Zudem können in der Interaktion auch gemeinsam weitere Bedeutungen erarbeitet werden, die auf ursprüngliche Deutungsmuster lediglich noch verweisen würden. Das Einbeziehen dieser Aushandlungsprozesse wäre für Studierende nützlich, um ein Interaktionsrepertoire kennen zu lernen, mit dem auf „Irritationen“ (Agiba 2016: 405f.), die durch Ausgangstexte verursacht werden, reagiert werden kann. Darüber hinaus brächten zweisprachige Beiträge in der Mutter- und der Zielsprache den Vorteil mit sich, dass wesentliches Vokabular unterschiedlicher germanistischer Forschungsrichtungen Jung-Akademikerinnen mitvermittelt wird und ihnen einen Einstieg in den internationalen Fachdiskurs ebnet.

\section{Fazit}

Betrachtet man die Zeiträume für die Entwicklung von Angeboten im Netz, dann scheint der richtige Zeitpunkt für einen Startschuss zur Beteiligung an der Wissensvermittlung im Netz durchaus gekommen, so dass in einer halben Generation Studierende für neue Lern- und Arbeitsmärkte ausgebildet werden können.

Der zweite wesentliche Aspekt für die Erarbeitung von Wissen über lern- und wissenschaftskulturelle Differenzen und Wege ihrer Bearbeitung ist der, dass die in der Germanistik gewonnenen Erkenntnisse, soweit sie die Kulturen des Lehrens und Lernens betreffen (und nicht das Fachwissen), auch anderen Fachdisziplinen vermittelt und verfügbar gemacht werden können, die sich weniger ex- 
plizit mit kulturellen Aspekten des Lernens und wissenschaftlichen Arbeitens auseinandersetzen.

Gemein ist allen Ansätzen, dass Wissen, auch theoretisches Wissen, Erfahrungs- und Handlungsräumen entspringt und somit regional gebunden ist. Welche Ausweitung der Begriff des Erfahrungs- und Handlungsraumes durch die Nutzung virtueller Forschungsräume erfährt, ist noch nicht abzusehen. Sicher ist jedoch, dass der analoge Raum mit seinen Praktiken jeweils partiell auch Eingang in virtuelle Räume finden wird und von daher die Notwendigkeit fortbestehen bleibt, in die virtuellen Räume hinein auch lebensweltliches Handlungswissen zu vermitteln.

Während in klassischen Lehr-Lern-Situationen die Lehrpersonen gemeinhin für die Kontextualisierung bzw. Rahmung von Wissen hauptsächlich verantwortlich waren, bringen es virtuelle Lernumgebungen mit sich, dass diese Ansprechpartner nicht per se existent sind. Um einen fachlich qualifizierten Zugang zum Fachwissen im Internet für Jung-Akademikerinnen zu ermöglichen, scheint es zum einen zweckmäßig, im Internet auch jene kommunikativen Vermittlungsräume zu etablieren, die analog verfügbar sind. Zum anderen aber sollten Formate erweitert oder ergänzt werden, um niedrigschwellige Einstiege in wissenschaftliche Diskurse zu eröffnen. Dazu zählt eine entsprechende Aufbereitung fachlichen Wissens, aber auch ein zusätzliches Wissen über Lern- und Arbeitskulturen. Deren Ziel ist es auch, Entstehung, Partizipation und Dissemination von Wissen einsichtig zu machen.

Die Formate im Internet sind für vielfältige Kontextualisierungen sowie interund transkulturelles Lernen bestens geeignet, da sie auf engstem Raum Mehrbezüglichkeit herzustellen vermögen, die sowohl spezifisch kulturelle Deutungsmuster als auch transkulturelle Vernetzungen offenlegen könnten.

Das offensichtliche Nebeneinander von Bedeutungen sorgte für die „Irritationen“, derer es bedarf, um kulturelle Lernprozesse anzuregen (vgl. Agiba 2016: 406).

\section{Literatur}

Agethen, Matthias et al. (2010/2015): Textpraxis - Digital Journal for Philology. Online: http:// www.uni-muenster.de/Textpraxis/en (01.03.2019).

Agiba, Sara (2016): „,Um uns zu [...] verwirren‘: Eine empirische Untersuchung zum kulturbezogenen Lernen“. In: Info DaF 43 (4), 401-416.

Altmayer, Claus (2004): Kultur als Hypertext. Zur Theorie und Praxis der Kulturwissenschaft im Fach Deutsch als Fremdsprache. München: iudicium.

Antos, Gerd (2005): „Die Rolle der Kommunikation bei der Konzeptualisierung von Wissensbegriffen“. In: Antos, Gerd; Wichter, Sigurd (Hrsg.): Wissenstransfer durch Sprache als gesellschaftliches Problem. Frankfurt a. M. etc.: Lang, 339-364 (Reihe Transferwissenschaften, 3). 
Antos, Gerd (2013): „Linguistische Resonanzforschung - Überlegungen zur Weiterentwicklung der Transferwissenschaften“. In: Ballod, Matthias; Weber, Tilo (Hrsg.): Autarke Kommunikation. Frankfurt a.M. etc.: Lang, 223-240 (Reihe Transferwissenschaften, 9).

Ballod, Matthias (2004): „Transfer oder Transformation? ,Wissen“ aus informationsdidaktischer Sicht“. In: Stenschke, Oliver; Wichter, Sigurd (Hrsg.): Theorie, Steuerung und Medien des Wissenstransfers. Frankfurt a.M. etc.: Lang, 103-113 (Reihe Transferwissenschaften, 2).

Borchert, Wolfgang (1949/1996): „Das Brot“. In: Wolfgang Borchert. Das Gesamtwerk. Reinbek: Rowohlt, 304-306.

Dewe, Bernd (2005): „Von der Wissenstransferforschung zur Wissenstransformation: Vermittlungsprozesse - Bedeutungsveränderungen“. In: Antos, Gerd; Wichter, Sigurd (Hrsg.): Wissenstransfer durch Sprache als gesellschaftliches Problem. Frankfurt a. M. etc.: Lang, 365-379 (Reihe Transferwissenschaften, 3).

Evers, Hans-Dieter; Gerke, Solvay (2003): Local and Global Knowledge: Social Science Research on Southeast Asia. Online: https://www.ioa.uni-bonn.de/institut-fuer-orient-und-asienwis senschaften/abteilungen/suedostasienwissenschaft/abteilung/working_18 (01.03.2019).

Evers, Hans-Dieter; Gerke, Solvay; Schweißhelm, Rebecca (2004): Malaysia, Singapur, Indonesien: Wege zur Wissensgesellschaft. Online: http://edoc.hu-berlin.de/evifa/article/evers-2 004-01-01/PDF/evers.pdf. (30.11.2015).

Feng, Xiaohu (1986): „Kurze Analyse von Wolfgang Borchert aufgrund seiner drei Werke“. In: Lehre der ausländischen Philologie 4. [Originaltitel chinesisch. In der Literaturliste der Seminararbeit erscheint die deutsche Übersetzung].

Franck, Julia (2002/2011): „Streuselschnecke“. In: Greese, Bettina (2011): Die Kurzgeschichte auf dem Weg ins 21. Jahrhundert. Braunschweig, Paderborn: Schöningh, 102.

Jannidis, Fotis et al. (2007): li-go. Online: http://www.li-go.de/definitionsansicht/ligostart.html (01.03.2019).

Kroner, Wolfgang; Wollf, Stephan (1984): „Auf der Suche nach der verlorenen Soziologie: Zu einigen Problemen empirischer Verwendungsforschung“. In: Soziale Welt 35 (4), 429-457.

Li, Shuangzhi (2009): „Dekodierung vs. Misreading: Überlegungen zur interkulturellen Rezeption der chinesischen Gegenwartslyrik in Deutschland“. In: Casper-Hehne, Hiltraud; Schweiger, Irmy (Hrsg.): Kulturelle Vielfalt in deutscher Literatur, Sprache und Medien. Göttingen: Universitätsverlag, 141-148.

Loenhoff, Jens (2014): „Kulturelle Differenz, interkulturelle Kommunikation und die Funktion des impliziten Wissens“. In: Meier, Simon; Rellstab, Daniel H.; Schiewer, Gesine L. (Hrsg.): Dialog und (Inter-)Kulturalität. Theorien, Konzepte, empirische Befunde. Tübingen: Narr, 25-41. Luhmann, Niklas (1987): „Die Richtigkeit soziologischer Theorie“. In: Merkur 41 (455), 36-49.

Mann, Thomas (1913/1992): Der Tod in Venedig. Frankfurt a. M.: Fischer Taschenbuch.

Machill, Katja (Red.); Wissenschaft im Dialog gGmbH (1999): Online: http://www.wissenschaftim-dialog.de/ (25.06.2017).

Middeke, Annegret (2009): „,Idial‘ für Germanisten und Slavisten: Interkultureller Dialog durch regionalisierte Lehrwerke“. In: Casper-Hehne, Hiltraud; Schweiger, Irmy (Hrsg.): Kulturelle Vielfalt deutscher Literatur, Sprache und Medien. Göttingen: Universitätsverlag, 45-51.

Neuser, Wolfgang (2013): Wissen begreifen. Zur Selbstorganisation von Erfahrung, Handlung und Begriff. Wiesbaden: Springer VS.

Otto, Gabriele E. (2018): Wissenschaftskulturen im Dialog - Wissenschaftsrezeption und Wissenschaftsmoderation im Zeitalter global verfügbarer Online-Publikationen. Berlin: Universitätsverlag der TU Berlin. 
Remus, Thérèse (2013): Literarisches Lernen sprachsensibel anleiten. Julia Francks Kurzgeschichte „Streuselschnecke“. München: GRIN. Online: http://www.grin.com/de/e-book/ 275636/literarisches-lernen-sprachsensibel-anleiten-julia-francks-kurzgeschichte (27.08.2017).

Sändig, Uta (2009): „Die Textsortenvariante ,literaturwissenschaftlicher Kurzessay“ und ihre Realisierung durch fremdsprachige Lerner“. In: Adamzik, Kirsten; Krause, Wolf-Dieter (Hrsg.): Text-Arbeiten. Textsorten im fremd-und muttersprachlichen Unterricht an Schule und Hochschule. 2. überarb. u. erw. Auflage. Tübingen: Narr, 155-171.

Schiewer, Gesine Lenore (2010): „Kooperation und Wettbewerb - Ein Widerspruch? Verständigung und Übersetzung im Blickfeld ökonomischer Emotionsforschung“. In: Zeitschrift für interkulturelle Germanistik 1/2010, 127-142.

Schlicht, Corinna; Marsch, Eva (Hrsg.) (2012): Von Geschichten, die ausziehen, das Leben zu erkunden: Einblicke in Julia Francks Erzählwelten. Mit einem Interview mit Julia Franck. Duisburg: Universitätsverlag Rhein-Ruhr.

Schmitt-Kaufhold, Angelika (2004): „Julia Franck: Streuselschnecke“. In: Kretzschmar, Bernd et al. (2004): Kompetenzorientierter Deutschunterricht - Kursstufe. Online: https:// lehrerfortbildung-bw.de/u_sprachlit/deutsch/gym/bp2004/fb2/02_klein/04_ue/02_bspl/ (01.03.2019).

Welsch, Wolfgang (1997): „Transkulturalität - Zur veränderten Verfassung heutiger Kultur“. In: Schneider, Irmela; Thomsen, Christian W. (Hrsg.): Hybridkultur: Medien, Netze, Künste. Köln: Wienand, 67-90.

Wichter, Sigurd (2005): „Wissenstransfer und Stereotypie. Zur Rolle von ungleichen Wissenskomplexen“. In: Antos, Gerd; Wichter, Sigurd (Hrsg.): Wissenstransfer durch Sprache als gesellschaftliches Problem. Frankfurt a.M. etc.: Lang, 403-418 (Reihe Transferwissenschaften, 3).

Winko, Simone; Arbeitsstelle für Theorie der Literatur an der Universität Göttingen (2007): Annotierte Bibliographie zur Literaturtheorie. Online: http://www.literaturtheorie.unigoettingen.de/glossar (01.03.2019).

\section{Internetquellen ohne Autorenangabe:}

Online: https://de.wikipedia.org/wiki/Das_Brot (01.03.2019).

Online: https://de.wikipedia.org/wiki/Eisbergmodell_(Literatur) (01.03.2019).

Online: https://de.wikipedia.org/wiki/Eisbergmodell (01.03.2019).

Online: https://de.wikipedia.org/wiki/Impliziter_Autor\#cite_ref-1 (10.01.2017).

Online: https://de.wikipedia.org/wiki/Julia_Franck (01.03.2019).

Online: https://de.wikipedia.org/wiki/Typologisches_Modell_der_Erz\%C3 \%A4hlsituationen (27.08.2017).

Online: https://de.wikipedia.org/wiki/Wolfgang_Borchert (27.08.2017).

Online: http://www.budapestopenaccessinitiative.org/ (01.03.2019).

Online: http://www.hausarbeiten.de/ (10. 01.2017) 


\section{Anhang: Internetquellen zur Entwicklung von frei verfügbarem wissenschaftlichem Wissen im Netz}

Arbeitsgruppe Open Access der Schwerpunktinitiative Digitale Information der Allianz der deutschen Wissenschaftsorganisationen. Online: https://www.bmbf.de/files/open-accessstrategien.pdf (26.03.2015).

Drotner, Kirsten (et al.) (2013): Humanities Scientific Committee Opinion Paper. Open Access Opportunities for the Humanities. Online: http://www.scienceeurope.org/ uploads/PublicDocumentsAndSpeeches/SE_Humanities_Paper_FIN.pdf, 4 (01.11.2015).

Georg-August-Universität Göttingen (2008): Open Access. Der freie Zugang zu wissenschaftlicher Information. Online: https://open-access.net (30.11.2015).

Helmholtz-Gemeinschaft (2008-2017): Schwerpunktinitiative „Digitale Information“. Online: http://www.allianzinitiative.de/handlungsfelder/open-access.html (21.09.2015).

Helmholtz-Gemeinschaft (2008-2017): Schwerpunktinitiative „Digitale Information“. Online: http://www.allianzinitiative.de/de/handlungsfelder/virtuelle-forschungsumgebung.html (19.11.2015).

Max-Planck-Gesellschaft zur Förderung der Wissenschaften e. V. Open Access Max-Planck-Gesellschaft (2003-2018): Online: http://openaccess.mpg.de/Berliner-Erklaerung (Zugriff am 26.03.2015).

\section{Biographische Angaben}

\section{Gabriele E. OTTO}

arbeitet seit 2016 als DAAD-Lektorin an der Shanghai International Studies University, P.R. of China. Arbeitsschwerpunkte: Literatur und Wissenschaftliches Arbeiten; Sprachunterricht und Lehrformate. Forschungsschwerpunkte: Wissenschaftsrezeption und Wissenschaftsmoderation im Zeitalter von Internet und Open Access (Forschungsstipendium des DAAD in Zusammenarbeit mit dem Lehrstuhl Deutsch als Fremd- und Fachsprache, TU Berlin); Narratologie. Weitere Lehrerfahrung: DAAD-Lektorat Universitas Indonesia, Jakarta, Republik Indonesien (2009-2014). 